\section{Surveillance for Influenza A 2009 H1N1 among Thai Healthcare Workers}

To the Editor-In Thailand, as of June 22, 2009, there were a total of 774 cases of influenza A 2009 HiN1. ${ }^{1}$ Notably, the majority of cases have occurred among persons who returned from countries with high attack rates and among school-aged children. Although the transmission of influenza A 2009 $\mathrm{H} 1 \mathrm{~N} 1$ in communities is well recognized, ${ }^{2}$ limited data are currently available with regard to the potential impact of influenza A $2009 \mathrm{H} 1 \mathrm{~N} 1$ transmission among healthcare workers (HCWs). From May 1 through June 22, 2009, surveillance for influenza A $2009 \mathrm{H} 1 \mathrm{Nl}$ was performed at Thammasat University Hospital in central Thailand. HCWs were screened for influenza A $2009 \mathrm{H} 1 \mathrm{~N} 1$ by means of rapid tests, polymerase chain reaction (PCR) assays, and viral culture if they had the onset of fever and influenza-like illness and one of the following: (1) recent travel to a country with persistent influenza A $2009 \mathrm{H} 1 \mathrm{Nl}$ activity per the World Health Organization $^{3} ;(2)$ contact with a suspected, probable, or confirmed influenza A $2009 \mathrm{H} 1 \mathrm{~N} 1$ case; or (3) contact with an ill HCW with suspected, probable, or confirmed influenza A 2009 H1N1.

During the study period, $54 \mathrm{HCWs}$ were screened for influenza A $2009 \mathrm{H} 1 \mathrm{Nl}$; $50 \mathrm{HCWs} \mathrm{(93 \% )} \mathrm{were} \mathrm{returning} \mathrm{from}$ countries with high H1N1 transmission activity, and $4(7 \%)$ had contact with an ill patient with suspected influenza $\mathrm{A}$ $2009 \mathrm{HIN1}$. All screened HCWs had negative results for the rapid tests and PCR assays. Notably, $1 \mathrm{HCW}(2 \%)$ developed fever and influenza-like illness on day 4 during his trip to the United States and received oseltamivir. He reported to the occupational health department 48 hours after returning to Thailand and had contact with $26 \mathrm{HCWs}$ in the hospital without wearing a face mask. The index $\mathrm{HCW}$ was quarantined, and all contacts were monitored for fever and influenza-like illness. The results of the rapid tests for the index $\mathrm{HCW}$ and all contacts were negative. However, the index HCW had a repeatedly persistent $\mathrm{H} 1$ band on PCR. Viral cultures obtained after the initiation of oseltamivir were negative. All contacts remained asymptomatic 14 days after exposure to the index case.

Our report has some implications for the heightened virulence and drug resistance anticipated for the second wave of the influenza A $2009 \mathrm{H} 1 \mathrm{Nl}$ pandemic. First and most importantly, surveillance of HCWs is essential. Implementing hospital-wide surveillance, early testing, and early empirical antiviral treatment may be crucial components of minimization of risk for transmission of influenza A 2009 H1N1 among HCWs. Second, surveillance definitions for HCWs should be modified according to the pandemic level and transmission activity. When influenza A H1N1 transmission activity is low in communities, exposure and transmission will likely continue among HCWs after travel to countries with high transmission activities and among HCWs with exposure to suspected, probable, or confirmed influenza A 2009 H1N1 cases. However, the appropriate surveillance definitions for HCWs when transmission is high in communities remain to be defined. Third, judicious antiviral use among HCWs will be important to limit the emergence of antiviralresistant influenza A $2009 \mathrm{H} 1 \mathrm{~N} 1$. Prescription of antiviral agents should be restricted to infectious diseases physicians or physicians responsible for public health after appropriate specimen collections. Because good knowledge and attitude toward influenza prevention does not necessarily imply good adherence to infection control practices, ${ }^{4}$ our study suggests the need to continually monitor infection control practices to help minimize influenza transmission during pandemics. In summary, additional studies are urgently needed to better define HCW surveillance strategies to minimize influenza transmission among HCWs in both resource-available and resource-limited countries.

\section{ACKNOWLEDGMENTS}

Financial support. Infectious Diseases and Infection Control Research Unit, Thammasat University Hospital, Pratumthani, Thailand (to A.A.); National Center for Genetic Engineering and Biotechnology, National Science and Technology Development Agency, Thailand (P-09-00435 to A.A.).

Potential conflicts of interest. Both authors report no conflicts of interest relevant to this article.

Anucha Apisarnthanarak, MD; Linda M. Mundy, MD, PhD

From the Division of Infectious Diseases, Thammasat University Hospital, Pratumthani, Thailand (A.A.); and LM Mundy, LLC, Bryn Mawr, Pennsylvania (L.M.).

Address reprint requests to Anucha Apisarnthanarak, MD, Division of Infectious Diseases, Thammasat University Hospital, Pratumthani, Thailand 12120 (anapisarn@yahoo.com).

Infect Control Hosp Epidemiol 2009; 30:1236

(C) 2009 by The Society for Healthcare Epidemiology of America. All rights reserved. 0899-823X/2009/3012-0018\$15.00. DOI: $10.1086 / 648659$

\section{REFERENCES}

1. Ministry of Public Health: Thailand. Available at: http://www.moph.go .th. Accessed July 22, 2009.

2. Shinde $\mathrm{V}$, Bridges $\mathrm{CB}$, Uyeki TM, et al. Triple-reassortant swine influenza A (H1) in humans in the United States, 2005-2009. N Engl J Med 2009; 360:2616-2625.

3. World Health Organization. Pandemic (H1N1) 2009. Available at: http: //www.who.int/csr/disease/swineflu/en/index.html. Accessed July 22, 2009.

4. Apisarnthanarak A, Phattanakeitchai P, Warren DK, Fraser VI. Impact of knowledge and positive attitudes about avian influenza (H5NI virus infection) on infection control and influenza vaccination practices of Thai healthcare workers. Infect Control Hosp Epidemiol 2008; 29:472-474. 